Gastroenterologe 2015 $\cdot 10: 265-267$

DOI 10.1007/s11377-015-1002-z

Online publiziert: 12. Juni 2015

(c) Springer-Verlag Berlin Heidelberg 2015
T. Berg' ${ }^{1}$ S. Zeuzem ${ }^{2}$

${ }^{1}$ Sektion Hepatologie, Klinik für Gastroenterologie und Rheumatologie, Department für Innere Medizin, Neurologie und Dermatologie, Universitätsklinikum Leipzig, Leipzig

${ }^{2}$ Medizinische Klinik 1, Klinikum der Goethe-Universität, Frankfurt a.M.

\title{
Effektive Kontrolle der HBV-Infektion und Heilung der HCV-Infektion durch direkt antivirale Substanzen
}

\author{
Welche Herausforderungen sind \\ jetzt noch zu erwarten?
}

Im Jahr 1999 wurde mit Lamivudin, einem nukleosidischen Inhibitor der reversen Transkriptase des HIV und der Hepatitis-B-Virus(HBV)-Polymerase, die erste direkt antiviral wirksame Substanz für die chronische Virushepatitis B zugelassen. Es hat sich innerhalb kürzester Zeit als Therapeutikum der ersten Wahl für HBV-infizierte Patenten etabliert. Im Unterschied zu der seit dem Jahr 1992 eingeführten Interferontherapie ermöglichte die Behandlung mit Lamivudin erstmals eine effektive Suppression der HBVReplikation bei nahezu allen Patienten. Es zeigte sich rasch, dass allein durch die Senkung der HBV-DNA („Viruslast“) klinische Endpunkte der akuten und chronischen HBV-Infektion günstig beeinflusst werden konnten. Besonders eindrucksvoll war der therapeutische Effekt bei schwerer Reaktivierung der HBV-Infektion nach Lebertransplantation, aber es ließ sich im Langzeitverlauf auch erstmals eine Regression der Fibrose und Reduktion der Rate hepatozellulärer Karzinome (HCC) nachweisen. Die mit der Selektion resistenter Virusvarianten assoziierten Durchbrüche der HBV-Replikation limitierte jedoch die Effizienz der Lamivudintherapie im Langzeitverlauf und führte zur Entwicklung von Polymeraseinhibitoren der 2. Generation mit verbessertem Wirkungs- und Resistenzprofil. Im Zuge dieser Dynamik antiviraler Therapiestra- tegien, die - wie im Folgenden noch dargestellt - in ganz besonderer Weise durch die Hepatitis-C-Forschung beflügelt wurde, hat sich ein hepatovirologischer Forschungsschwerpunkt innerhalb der Gastroenterologie entwickelt mit dem"Hepatovirologen“ als einem seiner Protagonisten.

Die kritische Evaluation der Langzeiteffizienz oraler HBV-Polymeraseinhibitoren ist Hauptthema des Beitrags von van Bömmel und Berg. Inzwischen sind, 15 Jahre nach der Einführung von Lamivudin, 5 nukleosidische bzw. nukleotidische HBV-Polymeraseinhibitoren (NUC, auch Nukleosid-/Nukleotidanaloga, NA, bezeichnet) zugelassen worden. Es ist aber v. a. die Entwicklung hochpotenter NUC, Entecavir und Tenofovir, die die Therapie der HBV-Infektion revolutioniert haben.

\section{1) Entecavir und Tenofovir haben die Therapie der HBV-Infektion revolutioniert}

Prospektive Langzeitverlaufsstudien über einen Zeitraum von mehr als 8 Jahren belegen die dauerhafte hohe antivirale Wirksamkeit und Sicherheit dieser Substanzen, die auch in der Monotherapie praktisch nicht zur Selektion resistenter HBV-Varianten führen. Ein Paradigmenwechsel in der Virologie, glaubte man doch bisher, dass nur durch die Kombination direkt antiviraler Substanzen die Resistenzentwicklung verhindert werden kann. Durch die NUC-vermittelte Kontrolle der HBVReplikation wird nicht nur die Progression der chronischen Hepatitis gestoppt, sondern auch - selbst in frühen Zirrhosestadien - eine Regression der Fibrose erreicht. Das persistierende Risiko der HCC-Entwicklung unter NUC-Therapie stellt ein ungelöstes Problem im Langzeitmanagement dar. Überwachungsstrategien sollten dabei an das individuelle Risiko angepasst werden, das relativ zuverlässig anhand von HCC-Risikoscores ermittelt werden kann. Bei allen Erfolgen der NUC-Therapie wird jedoch eine „Heilung“ der HBV-Infektion mit Verlust von HBsAg und Bildung von Anti-HBsAntikörpern nur sehr selten erreicht (etwa 10\%) und es lässt sich keine signifikante Zunahme dieses optimalen Endpunkts mit zunehmender Therapiedauer jenseits des 4. bis 5. Jahrs erreichen.

Nachdem inzwischen eine Kontrolle der HBV-Infektion mit Verhinderung klinischer Endpunkte in der überwiegenden Mehrzahl aller Patient erreicht werden kann, ist es das Ziel neuer therapeutischer Entwicklungen, die Heilungsraten (HBsAg-Serokonversion) zu steigern und damit häufiger einen Zustand zu generieren, bei dem die Therapie beendet werden kann. Aktuell werden v. a. unterschiedliche Kombinationstherapien aus pegylier- 
tem Interferon (PEG-IFN) plus NUC evaluiert. Diese liefern jedoch widersprüchliche Ergebnisse, wie in dem Beitrag von Petersen herausgestellt wird. Die Kombinationstherapie führt zwar initial zu einer stärkeren Reduktion der HBsAg-Konzentrationen, signifikante Effekte auf die Langzeit-HBsAg-Verlustrate konnten aber bislang noch nicht erzielt werden, sodass keiner dieser Ansätze Eingang in die Routine gefunden hat. Vergleichbar effektiv wie die „add-on" PEG-IFN-Strategie scheint auch das kontrollierte Absetzen der NUC-Therapie bei Pateinten ohne fortgeschrittene Erkrankung zu sein. Als Folge des Rebounds der HBV-Replikation nach Absetzen der NUC-Therapie erreichen einige Patienten einen HBsAg-Verlust. Dieser Ansatz wird in Deutschland über eine vom Bundesministerium für Bildung und Forschung geförderte Studie prospektiv evaluiert (STOP-NUC-Studie). Andere zukünftige Therapieoptionen sind von der klinischen Anwendung noch relativ weit entfernt. Hierzu zählen u. a. Entwicklungen im Bereich der therapeutischen Vakzine, Induktion des angeborenen Immunsystems durch Toll-likeRezeptor-Agonisten, HBV-entry- oder -capsid-Inhibitoren und Strategien, das HBV-Minichromosom (cccDNA) direkt $\mathrm{zu}$ „blockieren“.

Die chronische Hepatitis-C-Virus(HCV)-Infektion gehört weltweit zu den Hauptursachen für die Entwicklung einer Leberzirrhose und des HCC; sie ist mit einer signifikanten Steigerung der leberund auch nichtleberbezogenen Sterblichkeit assoziiert. Die globale HCV-RNAPrävalenz wird auf $1,1 \%$ geschätzt, das entspricht etwa 80 Mio. chronisch HCV-infizierten Patienten.

\section{\) Die globale HCV-RNA-Prävalenz wird auf $1,1 \%$ geschätzt}

Aufgrund dieser hohen HCV-Prävalenz wird mit einer signifikanten Steigerung der HCV-assoziierten Komplikationen in den kommenden Jahren gerechnet. In Deutschland beträgt die Zahl der HCVbedingten leberbezogenen Todesfälle aktuell 1200 und es wird im Jahr 2024 mit 1600 Sterbefällen pro Jahr gerechnet.
Seit der Entdeckung des HCV im Jahr 1989 konnte ein enormer therapeutischer Fortschritt für Patienten mit Hepatitis C erreicht werden. Inzwischen - 25 Jahre später - lassen sich interferonfrei durch eine 8- bis 12-wöchige Kombinationstherapie bestehend aus $2-3$ oralen direkt antiviral wirksamen Substanzen (DAA), mit oder ohne Ribavirin, Heilungsraten von über $90 \%$ erreichen. Die Effektivität ist selbst bei fortgeschrittener Fibrose und Zirrhose hoch, erfordert aber im Einzelfall eine Verlängerung der Therapiedauer auf 24 Wochen.

Eine sehr gute Übersicht zu den aktuell empfohlenen Therapieschemata in Abhängigkeit vom HCV-Typ liefert der Beitrag von Lange, Sarrazin und Zeuzem. Inzwischen sind in Deutschland 6 DAA (Sofosbuvir, Simeprevir, Daclatasvir, Ledipasvir, Paritaprevir, Ombitasvir, Dasabuvir) zugelassen, die interferonfrei in $\mathrm{Ab}$ hängigkeit vom HCV-Typ und bestimmten Patientencharakteristiken kombiniert eingesetzt werden. Die aktuellen Therapiestrategien folgen somit grundsätzlich dem Konzept einer individualisierten Therapie. Wesentliche Faktoren für die differenzialtherapeutischen Überlegungen sind v. a. der HCV-Typ und -Subtyp, Vortherapien (DAA-Resistenzen?), das Stadium der Lebererkrankung sowie Begleiterkrankungen (z.B. Niereninsuffizienz, Leberinsuffizienz) und Komedikationen aufgrund von möglichen Medikamenteninteraktionen. Therapeutische Herausforderungen stellen v. a. Patienten mit Zirrhose und HCV-Typ-3Infektion sowie Patienten mit dekompensierter Zirrhose vor Lebertransplantation und die Therapie der HCV-Reinfektion nach Transplantation dar. Diesem speziellen Thema widmet sich der Beitrag von Herzer und Gerken. Mit Ausnahme von Patienten mit HCV, Typ 3 können bei Patienten mit kompensierter Zirrhose Heilungsraten vergleichbar denen von Patienten ohne Zirrhose erreicht werden. Auch für diese Patienten ist eine 12-wöchige Therapie meist ausreichend, erfordert aber den zusätzlichen Einsatz von Ribavirin. Für Patienten mit dekompensierter Zirrhose liegen inzwischen Daten zur Wirksamkeit und Sicherheit der DAAKombinationstherapie vor. Heilungsraten von $80-90 \%$ nach 12 -wöchiger Therapie und über $90 \%$ bei 24 -wöchiger Therapiedauer verbunden mit einer signifikanten Verbesserung der Leberfunktion konnten erzielt werden. Die Induktion eines kompletten virologischen Responses vor Transplantation verhindert zudem effektiv die HCV-Reinfektion nach Organtransplantation. Langzeitverlaufsstudien müssen klären, bei wie vielen und welchen Patienten mit fortgeschrittenen Stadien der Lebererkrankung die Transplantation auch langfristig vermieden werden kann.

\section{》) Ein Screening zur \\ Detektion des hepatozellulären Karzinoms ist erforderlich}

Wie bei der HBV-Infektion ist ein HCCScreening erforderlich, da auch nach HCV-Eradikation bei Patienten mit Zirrhose im Langzeitverlauf in bis zu $10 \%$ der Fälle mit der Entwicklung eines HCC gerechnet werden muss. Bei der Therapie von Patienten mit HCV-Reinfektion nach Lebertransplantation müssen Medikamenteninteraktionen insbesondere mit den Kalzineurininhibitoren berücksichtigt werden. Die therapeutische Effizienz der DAA-Therapie ist jedoch auch bei dieser Patientenpopulation hoch und es lässt sich bereits jetzt schlussfolgern, dass die mit der HCV-Reinfektionshepatitis assoziierte erhöhte Sterblichkeit und Transplantatverlustrate bereits der Vergangenheit angehören.

Gelingt es aber nicht, die Therapierate zu steigern, wird trotz des hohen therapeutischen Fortschritts die Zahl der Patienten, die in den nächsten 10 Jahren an den Folgen der chronischen Hepatitis C sterben, nicht signifikant abnehmen. $\mathrm{Zu}$ dieser Schlussfolgerung kommen die Autoren Hardtke und Wedemeyer in ihrem Beitrag. Eine aktuelle Modellrechnung zeigt für Deutschland, dass mindestens $6000 \mathrm{~Pa}$ tienten mit HCV-Infektion pro Jahr identifiziert werden müssten, um die durch die HCV-Infektion verursachten Dekompensationen mittelfristig unter die Zahl von 1000 Fällen pro Jahr zu senken. Diese Daten zeigen eindrucksvoll, dass gerade jetzt mit der Zulassung der DAA-Therapie die Initiierung von HCV-Screeningprogrammen sinnvoll erscheint und dass auf- 
grund des ausgeprägten klinischen Nutzens mit Reduktion klinischer Endpunkte der chronischen HCV-Infektion auch langfristig Kosten gespart werden.

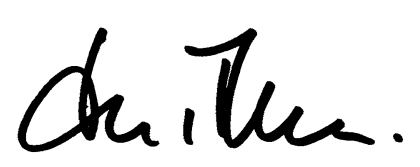

Prof. Dr. Thomas Berg

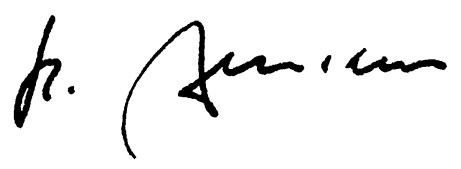

Prof. Dr. Stefan Zeuzem

\section{Korrespondenzadressen}

\section{Prof. Dr. T. Berg}

Sektion Hepatologie, Klinik für

Gastroenterologie und Rheumatologie, Department für Innere Medizin, Neurologie und Dermatologie, Universitätsklinikum Leipzig Liebigstraße 20, 04103 Leipzig thomas.berg@medizin.uni-leipzig.de

\section{Prof. Dr. S. Zeuzem}

Medizinische Klinik 1,

Klinikum der Goethe-Universität Theodor-Stern-Kai 7, 60590 Frankfurt a.M. zeuzem@em.uni-frankfurt.de

Interessenkonflikt. T. Berg gibt Beratungstätigkeiten für die Firmen Abbvie, Bristol-Myers Squibb, Merck, Novartis, Janssen und Roche an. S. Zeuzem gibt Beratungstätigkeiten für die Firmen Abbvie, Bristol-Myers Squibb, Gilead, Janssen, und Merck/MSD an.

\section{Galenus-von- Pergamon-Preis 2015}

\section{Arzneimittel-Innovationen sind im Rennen}

Alljährlich würdigt Springer Medizin Ärzte Zeitung herausragende Arzneimittel-Innovationen mit dem nationalen Galenus-von-Pergamon-Preis. Der Preis wird in den Kategorien „Primary Care", ,Specialist Care" und seit vergangenem Jahr in der Kategorie "Orphan Drugs" vergeben. Um den Preis können sich Arzneimittel-Innovationen bewerben, deren deutsche Zulassung und Markteinführung in der eingereichten Indikation nicht länger als drei Jahre zurückliegen. In diesem Jahr liegen 13 Bewerbungen für die Auszeichnung vor.

Über die Zuerkennung des Galenus-von-Pergamon-Preises entscheidet ein Kollegium von 14 unabhängigen Experten. Die Juroren bestimmen in einem Vorentscheid maximal fünf Kandidaten in jeder Kategorie, die an der Endrunde teilnehmen. Über die Zuerkennung des Galenus-Preises entscheidet das Kollegium am Tage der Preisverleihung, die im Rahmen einer festlichen Gala am 15. Oktober 2015 in Berlin stattfindet.

\section{Entyvio $^{\circledR}$ \\ (Vedolizumab)}

Mit Vedolizumab (Entyvio ${ }^{\circledR}$ ) von Takeda steht ein neues, darmselektives Wirkprinzip zur Therapie bei Colitis ulcerosa und Morbus Crohn zur Verfügung. Das Biologikum, das seit Juli 2014 am Markt ist, verhindert die Einwanderung von T-Lymphozyten in die Darmmukosa und dämpft somit gezielt die Entzündung im Darm. Damit unterscheidet sich die Substanz deutlich von den bisher eingesetzten Immunsuppressiva wie Azathioprin oder Anti-TNFa-Antikörpern, die alle systemisch wirken.

Vedolizumab wurde von der Europäischen Zulassungsbehörde EMA im Mai 2014 für die Behandlung erwachsener Patienten mit mittelschweren bis schweren aktiven Formen von Colitis ulcerosa und Morbus Crohn zugelassen, die auf konventionelle Therapien oder einen Anti-TNFa-Antikörper nur unzureichend oder nicht mehr ansprechen oder die Unverträglichkeiten dagegen aufweisen. Vedolizumab wird intravenös über 30 Minuten infundiert. Initial wird der Antikörper in einer Dosis von $300 \mathrm{mg}$ in Woche 0, 2 und 6 verabreicht. In der Erhaltungstherapie beträgt die Dosierung $300 \mathrm{mg}$ alle acht Wochen.

Quelle und weitere Infos: www.aerztezeitung.de 